Marquette University

e-Publications@Marquette

8-1-2007

\title{
X-ray Crystallographic Characterization of the Co(II)-substituted Tris-bound Form of the Aminopeptidase from Aeromonas proteolytica
}

Petra Munih

Brandeis University

Aaron Moulin

Brandeis University

Carin Stamper

Brandeis University

Brian Bennett

Marquette University, brian.bennett@marquette.edu

Dagmar Ringe

Brandeis University

See next page for additional authors

Accepted version. Journal of Inorganic Biochemistry, Vol. 101, No. 8 (August 2007): 1099-1107. DOI. (C) 2007 Elsevier Inc. Used with permission.

Brian Bennett was affiliated with the Medical College of Wisconsin at the time of publication. Richard Holz was affiliated with the Loyola University of Chicago at the time of publication. 
Authors

Petra Munih, Aaron Moulin, Carin Stamper, Brian Bennett, Dagmar Ringe, Gregory A. Petsko, and Richard C. Holz 


\section{Marquette University}

\section{e-Publications@Marquette}

\section{Physics Faculty Research and Publications/College of Arts and Sciences}

This paper is NOT THE PUBLISHED VERSION; but the author's final, peer-reviewed manuscript. The published version may be accessed by following the link in the citation below.

Journal of Inorganic Biochemistry, Vol. 10a1, No. 8 (August 2007): 1099-1107. DOI. This article is (C) Elsevier and permission has been granted for this version to appear in e-Publications@Marquette. Elsevier does not grant permission for this article to be further copied/distributed or hosted elsewhere without the express permission from Elsevier.

\section{X-ray crystallographic characterization of the Co(II)-substituted Tris-bound form of the aminopeptidase from Aeromonas proteolytica}

Petra Munih

Program in Biophysics and Structural Biology, Rosenstiel Basic Medical Sciences Research Center, Brandeis University, 415 South Street, Waltham, MA

Departments of Biochemistry and Chemistry, Rosenstiel Basic Medical Sciences Research Center, Brandeis University, 415 South Street, Waltham, MA

Aaron Moulin

Program in Biophysics and Structural Biology, Rosenstiel Basic Medical Sciences Research Center, Brandeis University, 415 South Street, Waltham, MA

Departments of Biochemistry and Chemistry, Rosenstiel Basic Medical Sciences Research Center, Brandeis University, 415 South Street, Waltham, MA

\section{Carin C. Stamper}

Program in Biophysics and Structural Biology, Rosenstiel Basic Medical Sciences Research Center, Brandeis University, 415 South Street, Waltham, MA

Departments of Biochemistry and Chemistry, Rosenstiel Basic Medical Sciences Research Center, Brandeis University, 415 South Street, Waltham, MA 


\section{Brian Bennett}

Department of Physics, Marquette University, Milwaukee, WI

National Biomedical EPR Center, Department of Biophysics, Medical College of Wisconsin, Milwaukee, WI

\section{Dagmar Ringe}

Program in Biophysics and Structural Biology, Rosenstiel Basic Medical Sciences Research Center, Brandeis University, 415 South Street, Waltham, MA

Departments of Biochemistry and Chemistry, Rosenstiel Basic Medical Sciences Research Center, Brandeis University, 415 South Street, Waltham, MA

\section{Gregory A. Petsko}

Program in Biophysics and Structural Biology, Rosenstiel Basic Medical Sciences Research Center, Brandeis University, 415 South Street, Waltham, MA

Departments of Biochemistry and Chemistry, Rosenstiel Basic Medical Sciences Research Center, Brandeis University, 415 South Street, Waltham, MA

\section{Richard C. Holz}

Department of Chemistry, Marquette University, Milwaukee, WI

Department of Chemistry, Loyola University-Chicago, 1068 West Sheridan Road, Chicago, IL

\section{Abstract}

The $\underline{X}$-ray crystal structure of the Co(II)-loaded form of the aminopeptidase from Aeromonas proteolytica ([CoCo(AAP)]) was solved to $2.2 \AA$ resolution. [CoCo(AAP)] folds into an $\alpha / \beta$ globular domain with a twisted $\underline{\beta-}$ sheet hydrophobic core sandwiched between $\alpha$-helices, identical to [ZnZn(AAP)]. Co(II) binding to AAP does not introduce any major conformational changes to the overall protein structure and the amino acid residues ligated to the dicobalt(II) cluster in [CoCo(AAP)] are the same as those in the native $\mathrm{Zn}(\mathrm{II})$-loaded structure with only minor perturbations in bond lengths. The $\mathrm{Co}(\mathrm{II})-\mathrm{Co}(\mathrm{II})$ distance is $3.3 \AA$. Tris(hydroxymethyl)aminomethane (Tris) coordinates to the dinuclear Co(II) active site of AAP with one of the Tris hydroxyl oxygen atoms (O4) forming a single oxygen atom bridge between the two Co(II) ions. This is the only Tris atom coordinated to the metals with Co1-O and Co2-O bonds distances of 2.2 and $1.9 \AA$, respectively. Each of the $\mathrm{Co}$ (II) ions resides in a distorted trigonal bipyramidal geometry. This important structure bridges the gap between previous structural and spectroscopic studies performed on AAP and is discussed in this context.

\section{Keywords}

Hydrolysis, Aminopeptidase, Zinc, Cobalt, EPR, Electronic absorption, X-ray crystallography, Tris

\section{Introduction}

Hydrolases that contain co-catalytic metallo-active sites catalyze diverse reactions such as the degradation of DNA, RNA, phospholipids, and polypeptides [1], [2], [3], [4]. They are therefore key players in carcinogenesis, tissue repair, protein maturation, hormone level regulation, cell-cycle control, and protein degradation processes. In addition, co-catalytic metallohydrolases are involved in the degradation of agricultural neurotoxins, urea, $\beta$-lactam containing antibiotics, and several phosphorus(V) materials used in chemical weaponry [5], [6], [7]. The majority of co-catalytic metallohydrolases are Zn(II) dependent enzymes; however, several can utilize a wide range of first row transition metal ions. Regardless of the active site metal ion identity, the structural properties (e.g. coordination geometry and ligand type) regulate their Lewis acidity, which in turn, controls the level of hydrolytic activity. Possible roles for one or both metal ions in hydrolases that contain co- 
catalytic active sites include: (i) binding and positioning substrate, (ii) binding and activating a water molecule to yield an active site hydroxide nucleophile, and/or (iii) stabilizing the transition state of the hydrolytic reaction.

The importance of understanding the mechanism of action of co-catalytic metallohydrolases is underscored by the observations that the naturally occurring peptide analog inhibitor, bestatin, significantly decreases HIV infection in males by inhibiting aminopeptidase activity [8].

A common practice when studying $\mathrm{Zn}(\mathrm{II})$ containing enzymes is to substitute the magnetically and spectroscopically silent $\mathrm{d}^{10} \mathrm{Zn}$ (II) ion with Co(II) [9]. Co(II) appears to be an excellent replacement probe of $\mathrm{Zn}$ (II) centers, because it has a similar ionic radius to that of $Z n(I I)(0.74$ vs. $0.75 \AA)$, has seven d-electrons, and is paramagnetic $(S=3 / 2)$. Moreover, ligand-field stabilization energies (LFSE's) for $\mathrm{Zn}(\mathrm{II})$ in both octahedral $\left(O_{\mathrm{h}}\right)$ and tetrahedral $\left(T_{\mathrm{d}}\right)$ geometries are zero, suggesting that neither structure is favored. However, based on X-ray crystallographic data, $\mathrm{Zn}$ (II) clearly favors $T_{\mathrm{d}}$ geometries [10]. The reason is that the $4 \mathrm{~s}$ and $4 \mathrm{p}$ orbitals are easily accessible as electron-pair acceptors, providing an $\mathrm{sp}^{3}$-type center, while the $5 \mathrm{~d}$ orbitals are higher in energy making the formation of $O_{\mathrm{h}}$ or trigonal bipyramidal (TBP) complexes slightly less favorable. LFSE's for Co(II) favor $T_{\mathrm{d}}$ complexes by $2 / 5 \Delta_{0}$, suggesting that $\mathrm{Co}$ (II) ions also adopt similar coordination geometries to $\mathrm{Zn}$ (II) [11]. Spectroscopic and kinetic studies on Co(II)-substituted Zn(II) metalloproteases have provided much of the mechanistic information available on this class of enzymes. For example, the $\mathrm{Zn}(\mathrm{II})$-containing metalloaminopeptidase from Aeromonas proteolytica (AAP), has been substituted with Co(II) in vitro providing a hyperactive enzyme [12], [13]. Similar results have been obtained on a wide variety of Zn(II) metalloproteases including carboxypeptidase A and thermolysin [9], [14].

Despite their ubiquity and the considerable structural information available on $\mathrm{Zn}$ (II) forms of metallohydrolases containing co-catalytic active sites, little is known about how these structural motifs relate to the Co(II)substituted forms of these enzymes [15], [16], [17]. Therefore, we have solved the X-ray crystal structure of the Co(II)-substituted form of AAP ([CoCo(AAP)]) to $2.2 \AA$ resolution, in order to understand the structural aspects of Co(II) binding to AAP. This important structure bridges the gap between previous structural and spectroscopic studies performed on AAP.

\section{Materials and methods}

\subsection{Enzyme purification}

All chemicals used in this study were purchased commercially and were of the highest quality available. The aminopeptidase from $A$. proteolytica was purified from a stock culture kindly provided by Professor Céline Schalk. Cultures were grown according to the previously published procedure [18] with minor modifications [19] to the growth media. Purified enzyme was stored at $77 \mathrm{~K}$ until needed.

\subsection{Spectrophotometric assay}

AAP activity was measured by the method of Prescott and Wilkes [18] as modified by Baker et al. [20]. In this assay, the hydrolysis of $0.5 \mathrm{mM}$ I-leucine $p$-nitroanilide (I-pNA) (10 mM Tricine, $\mathrm{pH} 8.0$ ) was measured spectrophotometrically at $25^{\circ} \mathrm{C}$ by monitoring the formation of $p$-nitroaniline. The extent of hydrolysis was calculated by monitoring the increase in absorbance at $405 \mathrm{~nm}\left(\Delta \varepsilon_{405}\right.$ value of $p$-nitroaniline of $\left.10,800 \mathrm{M}^{-1} \mathrm{~cm}^{-1}\right)$. One unit is defined as the amount of enzyme that releases $1 \mu \mathrm{mol}$ of $p$-nitroaniline at $25^{\circ} \mathrm{C}$ in $60 \mathrm{~s}$. Depletion of enzyme-bound zinc or cobalt was prevented by the addition of $0.1 \mathrm{mM} \mathrm{ZnSO}_{4}$ or $\mathrm{CoCl}_{2}$ to the buffer. The specific activity of purified Zn(II)-bound AAP was typically found to be 120 units per $\mathrm{mg}$ of enzyme. This value is identical to that reported by Prescott and Wilkes [18]. Enzyme concentrations were determined from the absorbance at $280 \mathrm{~nm}$ with the value $\varepsilon_{280}=41,800 \mathrm{M}^{-1} \mathrm{~cm}^{-1}$ [21]. The accuracy of this value was checked by the Edelhoch method [22], [23], [24] using a 5:13:2 molar ratio mixture of $\mathrm{N}$-acetyl-I-tryptophanamide, Gly-Tyr-amide, and Icysteine, respectively, to model AAP. The molar absorptivity determined from this method, $\varepsilon_{280}=43,950 \mathrm{M}^{-1} \mathrm{~cm}^{-1}$ for AAP solubilized in $6 \mathrm{M}$ guanidine hydrochloride, was in excellent agreement with the previously reported value by Prescott et al. [21]. 


\subsection{Spectroscopic measurements}

All spectrophotometric measurements were performed on a Shimadzu UV-3101PC spectrophotometer equipped with a constant temperature holder and a Haake (Type 423) constant temperature circulating bath. The use of $200 \mu \mathrm{L}, 1 \mathrm{~cm}$ path-length microcuvettes (QS, Hellma) stoppered with rubber septa facilitated the recording of the optical spectra under anaerobic conditions.

\subsection{Crystallization}

Co(II)-substituted AAP was crystallized using the crystallization conditions reported for the native enzyme [25]. Briefly, purified AAP $(10 \mathrm{mg} / \mathrm{mL})$ in $10 \mathrm{mM}$ Tris $\mathrm{pH} 8.0,10 \mathrm{mM} \mathrm{KSCN}, 0.4 \mathrm{M} \mathrm{NaCl}$ was crystallized by vapor diffusion using $100 \mathrm{mM}$ Tris pH 8.0, $100 \mathrm{mM} \mathrm{KSCN}$, and $4.5 \mathrm{M} \mathrm{NaCl}$ as the precipitating solution. The crystals were left to form and grow in an anaerobic chamber at $4{ }^{\circ} \mathrm{C}$ to avoid Co(II) oxidation into Co(III). Crystals, much smaller than for native AAP $(0.7 \mathrm{~mm} \times 0.4 \mathrm{~mm} \times 0.4 \mathrm{~mm}$ [28]), with dimensions $0.3 \mathrm{~mm} \times 0.2 \mathrm{~mm} \times 0.2 \mathrm{~mm}$ were obtained in a few weeks. This is significantly longer than for native AAP which routinely needs only two days to crystallize. The [CoCo(AAP)] crystals were shown to be isomorphous with the native ones. The crystals belong to space group P6 122 with the following unit cell dimensions: $a=b=106.2 \AA, c=96.7 \AA, \alpha=6=90^{\circ}, \gamma=120^{\circ}$. As with native AAP, there is one monomer per asymmetric unit.

\subsection{Data collection and processing}

Diffraction data were collected at the APS - BioCARS, Sector 14 BM-D Station, on an ADSC Quantum1 CCD detector. The crystal to detector distance was $90 \mathrm{~mm}$ and the exposure time $10 \mathrm{~s}$. The data were collected with an oscillation step size of $1.0^{\circ}$ on a single crystal. One crystal was used for the entire data collection period. The diffraction data were integrated and scaled using Denzo and Scalepack [26]. The data are highly redundant, with $98.6 \%$ of the reflections measured seven times or more and $58.7 \%$ of the observed reflections measured more than 19 times. With the data so redundant, the $R_{\text {merge }}$ values are artificially raised since the correlation between $I$ and $l_{\text {avg }}$ becomes lower with greater redundancy. The overall $R_{\text {merge }}$ is $12.0 \%$ and in the highest resolution shell $(2.23-2.15 \AA) R_{\text {merge }}$ is $74.3 \%$. The $l_{\text {avg }} / \sigma(I)$ avg is 3.6 in the outer resolution shell. Data collection and refinement statistics are provided in Table 1.

Table 1. Data collection and refinement statistics

\begin{tabular}{|c|c|}
\hline \multicolumn{2}{|l|}{ Crystal data } \\
\hline Space group & $P 6_{1} 22$ \\
\hline \multirow[t]{3}{*}{ Unit cell parameters $(\AA ̊)$} & $a=106.2$ \\
\hline & $b=106.2$ \\
\hline & $c=96.7$ \\
\hline \multicolumn{2}{|l|}{ Data processing } \\
\hline No. of reflections, observed & 833,939 \\
\hline No. of reflections, unique & 18,112 \\
\hline Cutoff $(I / \sigma)$ & 0 \\
\hline$R_{\text {merge, }}$ a overall (\%) & 12.0 \\
\hline Completeness, overall (\%) & 99.9 \\
\hline Highest resolution shell ( $\AA$ ) & $2.23-2.15$ \\
\hline$R_{\text {merge, }}$ a outer shell (\%) & 74.3 \\
\hline Completeness, outer shell (\%) & 99.9 \\
\hline \multicolumn{2}{|l|}{ Model refinement } \\
\hline Resolution range $(\AA ̊)$ & $30-2.15$ \\
\hline Cutoff $(F / \sigma F)$ & 0 \\
\hline
\end{tabular}




\begin{tabular}{|l|l|}
\hline$R$-factorb $(\%)$ & 22.4 \\
\hline No. of reflections & 16,585 \\
\hline$R_{\text {free }}$ (for 2053 reflections; \%) & 27.3 \\
\hline No. of protein atoms & 2211 \\
\hline No. of cobalt ions & 2 \\
\hline No. of Tris atoms & 8 \\
\hline No. of water molecules & 85 \\
\hline$B$-factor model & Individual \\
\hline$R M S D$ from ideality & \\
\hline Bond lengths ( $\AA$ ) & 0.008 \\
\hline Bond angles $\left({ }^{\circ}\right)$ & 1.40 \\
\hline Improper angles $\left(^{\circ}\right)$ & 0.96 \\
\hline Dihedral angles $\left({ }^{\circ}\right)$ & 22.7 \\
\hline Residues in most favored positions $\underline{c}(\%)$ & 84.9 \\
\hline
\end{tabular}

$\begin{array}{ll}\text { a. } & R_{\text {merge }}=\sum\left|I_{\text {obs }}-I_{\text {avg }}\right| / \sum I_{\text {avg }} \\ \text { b. } & R \text {-factor }=\sum\left|F_{\text {obs }}-F_{\text {calc }}\right| / \sum\left|F_{\text {obs }}\right| \\ \text { c. } & \text { As determined by the program PROCHECK. }\end{array}$

\subsection{Structure solution and refinement}

Since the enzyme crystals were isomorphous with those of the native enzyme, phases were derived from a previously solved structure (accession code 1AMP) [25]. In this process, water molecules were omitted from the original coordinate file. All refinement procedures were carried out using the software package CNS [27]. A test data set to calculate the $R_{\text {free }}$ [28] was made using $10 \%$ (1634 out of 16585) of the unique reflections. The original coordinate file was modified to create the starting model for refinement. All of the water molecules as well as the two $\mathrm{Zn}^{2+}$ cations with the side chains of their protein ligands were omitted to avoid introducing any bias from the native zinc coordination spheres. This initial model was subjected to a rigid body refinement using reflections in the 20.0-4.0 $\AA$ range. Subsequently, four rounds of minimization were carried out using incrementally higher resolution data; 8-3.0 $\AA, 2.8 \AA, 2.5 \AA$ and 2.15 (2.2) $\AA$. This resulted in an $R_{\text {free }}$ of $42.3 \%$ and a working $R$-factor of $34.8 \%$. Electron density maps with amplitudes $2 F_{\text {obs }}-F_{\text {calc }}$ and $F_{\text {obs }}-F_{\text {calc }}$ were then calculated and showed clear electron density in the active site for two Co(II) ions. At this point both $\mathrm{Co}(\mathrm{II})$ ions were modeled into the density using $\mathrm{O}(40)$. Subsequent manual manipulations of all atomic models were performed in the program $O$. After minimization and group $B$-factor refinement, the $R_{\text {free }}$ dropped to $36.7 \%$ and the working $R$-factor to $28.8 \%$.

In subsequent rounds of model building, minimization and individual $B$-factor refinement, water molecules were added to the model using the WATERPICK protocol in the CNS program. A constellation of waters was placed into the active site density initially, but upon careful inspection of the electron density shape and the network the waters formed, it became clear that another molecule might be bound in the active site. Based on the mother liquor solution used during crystallization, the only reasonable candidate was a Tris molecule. Therefore, the four water molecules were removed from the active site and the model was subjected to another round of minimization and individual $B$-factor refinement. $2 F_{\text {obs }}-F_{\text {calc }}$ as well as $F_{\text {obs }}-F_{\text {calc }}$ electron density maps were calculated and showed distinct electron density in the active site "coordinating" to the two metals. To remove any bias from the existent model, simulated annealing omit maps were calculated. In the first of the two sets of maps, only the active site ligand was omitted from the calculation. In the second, the whole active site was omitted. This included the two Co(II) cations, the side chains of their coordinating protein ligands and the postulated Tris molecule in the active site. The resulting maps are very similar to the electron density maps calculated on an "empty" active site mentioned above, indicating that the density observed adjacent to the dicobalt(II) center does not belong to water molecules. At different contour levels, the electron density 
appeared not to be completely explained by a single Tris molecule so we attempted to interpret it in terms of two modes of Tris coordination to the bimetal core. We also explored an interpretation in which a single Tris binding mode coexists with a small amount of unliganded enzyme with a water molecule bound between the two cobalt ions. Refinement of the structure with a single-bound Tris conformation, at $70 \%$ occupancy, yielded difference electron density which is more consistent with the latter interpretation. Because the resolution of this structure is only $2.2 \AA$, we elected not to build a second protein model with the water bound between the metals and refine partial occupancies for the two structures, as the ratio of observations to parameters is too low to allow this to be done accurately. The final model contains 291 protein amino acids, two cobalt cations, 8 Tris atoms, and 85 water molecules refined to a working $R$-factor of $22.4 \%$ and an $R_{\text {free }}$ of $27.3 \%$. The Luzzati plot estimated coordinate position error at this resolution is $0.3 \AA$.

\section{Results}

The X-ray crystal structure of [CoCo(AAP)] was solved to 2.2 A resolution (Fig. 1). [CoCo(AAP)] folds into an $\alpha / \beta$ globular domain with a twisted $\beta$-sheet hydrophobic core sandwiched between $\alpha$-helices, similar to [ZnZn(AAP)] [25]. The rms deviations for the 291 structurally equivalent $C \alpha$ atoms between the Co(II)-substituted and other crystallographically characterized Zn(II) AAP enzymes are 1.36, 2.32, and $2.09 \AA$ for [ZnZn(AAP)]-BuBA [28], [ZnZn(AAP)]-LPA [29], and [ZnZn(AAP)]-Tris [30], respectively. Therefore, Co(II) binding to AAP does not introduce any major conformational changes to the overall protein structure. Similar to [ZnZn(AAP)], a well defined hydrophobic pocket resides adjacent to the dinuclear Co(II) active site and is made up of Leu155, Met180, Ile193, Cys223, Tyr225, Cys227, Met242, Phe244, Phe248, Tyr251, and Ile255. The two Cys residues that reside at the back of the hydrophobic pocket (Cys223 and Cys227) form a disulfide bond. The amino acid residues ligated to the dicobalt(II) cluster in [CoCo(AAP)] are identical to those in the native $\mathrm{Zn}$ (II)-loaded structure with only minor perturbations in bond lengths (Table 2). The Co(II)-Co(II) distance is $3.3 \AA$.

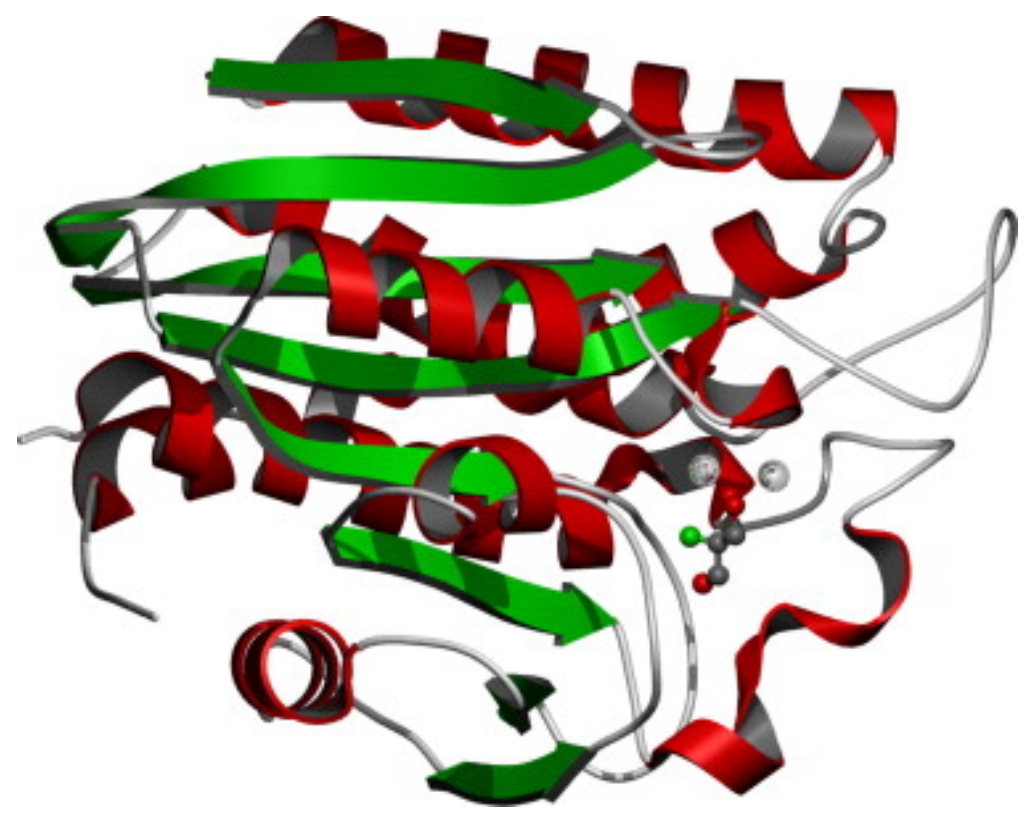

Fig. 1. The overall fold of the [CoCo(AAP)] enzyme with the two-bound $\mathrm{Co}(\mathrm{II})$ ions and the bound ligand shown.

Table 2. Selected distances (in $\AA$ ) for various AAP complexes 


\begin{tabular}{|c|c|c|c|c|c|c|c|}
\hline & & $\begin{array}{c}\text { Distance } \\
\text { (Å) }\end{array}$ & & & & & \\
\hline & & $\begin{array}{c}\text { Native } \\
{[25]}\end{array}$ & $\begin{array}{c}\text { AAP-BuBA } \\
{[28]}\end{array}$ & $\begin{array}{c}\text { AAP-LPA } \\
{[29]}\end{array}$ & $\begin{array}{c}\text { AAP-Bestatin } \\
{[37]}\end{array}$ & $\begin{array}{c}\text { AAP-Tris } \\
{[30]}\end{array}$ & $\begin{array}{l}\text { CoCoAAP- } \\
\text { Tris }\end{array}$ \\
\hline Zn1/Co1 & $\mathrm{Zn} 2$ & 3.5 & 3.3 & 3.9 & 3.5 & 3.5 & 3.3 \\
\hline \multirow[t]{13}{*}{ Zn1/Co1 } & $\begin{array}{l}\text { Asp117 } \\
\text { OD2 }\end{array}$ & 2.1 & 2.2 & 1.9 & 2.0 & 2.0 & 2.0 \\
\hline & $\begin{array}{l}\text { Glu152 } \\
\text { OE1 }\end{array}$ & 2.0 & 2.2 & 2.0 & 2.3 & 2.1 & 2.1 \\
\hline & $\begin{array}{l}\text { Glu152 } \\
\text { OE2 }\end{array}$ & 2.4 & 2.6 & 2.4 & 2.2 & 2.4 & 2.3 \\
\hline & His256 NE & 2.3 & 2.3 & 2.1 & 2.1 & 2.1 & 1.8 \\
\hline & $\mathrm{H}_{2} \mathrm{O}$ & 2.3 & & & & & \\
\hline & BuBA O1 & & 2.5 & & & & \\
\hline & BuBA 02 & & 2.7 & & & & \\
\hline & LPA 01 & & & 1.9 & & & \\
\hline & LPA 03 & & & 3.4 & & & \\
\hline & $\begin{array}{l}\text { Bestatin } \\
01\end{array}$ & & & & 2.1 & & \\
\hline & $\begin{array}{l}\text { Bestatin } \\
\mathrm{O} 2\end{array}$ & & & & 2.3 & & \\
\hline & Tris $\mathrm{O} 4$ & & & & & 2.2 & 2.2 \\
\hline & Tris N1 & & & & & 2.1 & 4.8 \\
\hline \multirow[t]{13}{*}{$\mathrm{Zn} 2 / \mathrm{Co} 2$} & $\begin{array}{l}\text { Asp117 } \\
\text { OD1 }\end{array}$ & 2.0 & 2.1 & 2.0 & 2.0 & 2.0 & 2.1 \\
\hline & $\begin{array}{l}\text { Asp179 } \\
\text { OD1 }\end{array}$ & 2.1 & 2.2 & 2.2 & 2.2 & 2.1 & 2.0 \\
\hline & $\begin{array}{l}\text { Asp179 } \\
\text { OD2 }\end{array}$ & 2.3 & 2.5 & 2.4 & 2.4 & 2.3 & 2.2 \\
\hline & His97 NE & 2.3 & 2.2 & 2.2 & 2.2 & 2.0 & 2.0 \\
\hline & $\mathrm{H}_{2} \mathrm{O}$ & 2.3 & & & & & \\
\hline & BuBA O2 & & 3.0 & & & & \\
\hline & BuBA 01 & & 4.4 & & & & \\
\hline & LPA 03 & & & 2.3 & & & \\
\hline & LPA NH & & & 2.1 & & & \\
\hline & $\begin{array}{l}\text { Bestatin } \\
01\end{array}$ & & & & 1.9 & & \\
\hline & $\begin{array}{l}\text { Bestatin } \\
\mathrm{NH}_{2}\end{array}$ & & & & 2.3 & & \\
\hline & Tris $\mathrm{O} 4$ & & & & & 2.0 & 1.9 \\
\hline & Tris $\mathrm{O5}$ & & & & & 2.1 & 4.0 \\
\hline \multirow{3}{*}{$\begin{array}{l}\text { Glu151 } \\
\text { OE1 }\end{array}$} & LPA O3 & & & 2.4 & & & \\
\hline & $\begin{array}{l}\text { Bestatin } \\
\mathrm{O} 1\end{array}$ & & & & 3.0 & & \\
\hline & Tris $\mathrm{O} 4$ & & & & & 2.7 & 3.9 \\
\hline $\begin{array}{l}\text { Glu151 } \\
\text { OE2 }\end{array}$ & LPA O3 & & & 2.8 & & & \\
\hline
\end{tabular}




\begin{tabular}{|l|l|l|l|l|l|}
\hline Bestatin & & 3.3 & \\
NH & & & & \\
\hline Tris O4 & & & & 4.4 \\
\hline Tris O5 & & & & 3.1 \\
\hline
\end{tabular}

After several rounds of refinement it became clear that in this case the elongated electron density cloud in the active site region did not belong to a constellation of water molecules as suggested by Chevrier et al. [25] but instead comprised a molecule of Tris, which was used as the crystallization buffer. Tris ligation was deduced based on the shape of the cloud and the composition of the crystallization solution. Moreover, the simulated annealing omit map with both Co(II) cations and all of their ligands removed indicated that a Tris molecule binds to [CoCo(AAP)]. Due presumably to partial occupancy and weak binding of Tris to the dinuclear Co(II) center, the observed density is not completely unambiguous, but does allow for an acceptable fit of the Tris molecule in a single orientation.

In this Tris binding mode, one of the hydroxyl oxygen atoms of Tris (O4) forms a single oxygen atom bridge between the two Co(II) ions (Fig. 2). This is the only Tris atom coordinated to the metals with Co1-O4 and Co2$\mathrm{O} 4$ bond distances of 2.2 and $1.9 \AA$, respectively (Fig. 3, Fig. 4). Each of the Co(II) ions resides in a distorted TBP geometry with the $N^{\varepsilon}$ nitrogen of His 256 and the bridging hydroxyl oxygen atom (O4) of Tris making up the axial positions for $\mathrm{Co} 1$. For $\mathrm{Co} 2$, the $N^{\varepsilon}$ nitrogen of His97 resides in an axial position along with the bridging hydroxyl oxygen atom (O4) of Tris. Glu152 is coordinated in an asymmetric bidentate fashion to $\mathrm{Co} 1$ with the additional oxygen atom providing the fifth ligand at a distance of $2.3 \AA$. Similarly, Asp179 is coordinated to Co2 in an asymmetric bidentate fashion with the second oxygen atom residing $2.2 \AA$ from the metal center. Following refinement of Tris in this conformation, the $2 F_{\text {obs }}-F_{\text {calc }}$ electron density map was relatively clean, although not as sharp as might be expected at this resolution if the inhibitor were tightly bound. An $F_{\text {obs }}-F_{\text {calc }}$ residual electron density map shows several small but significant features in the neighborhood of the Tris (data not shown). We attempted to interpret them in terms of a second Tris conformation, but no unique interpretation was possible and the residual density is not convincing in terms of such a conformation. The most likely interpretation of the residual electron density is some motional freedom of the single Tris conformation, especially in its terminal 06 oxygen atom, combined with a small amount of unliganded enzyme, containing a water molecule between the two cobalt ions, in the crystal lattice.
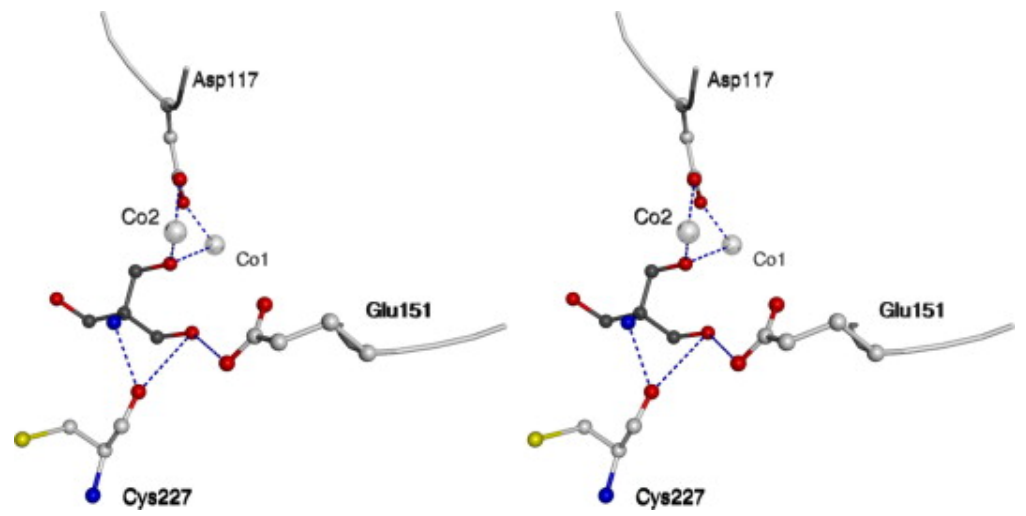

Fig. 2. Stereo figure of the active site region of [CoCo(AAP)] with $\underline{T r i s}$ bound. The Tris molecule is colored dark gray (see Fig. 3), while the protein ligand is colored light gray. The bridging oxygen atom of Tris is 04 . 


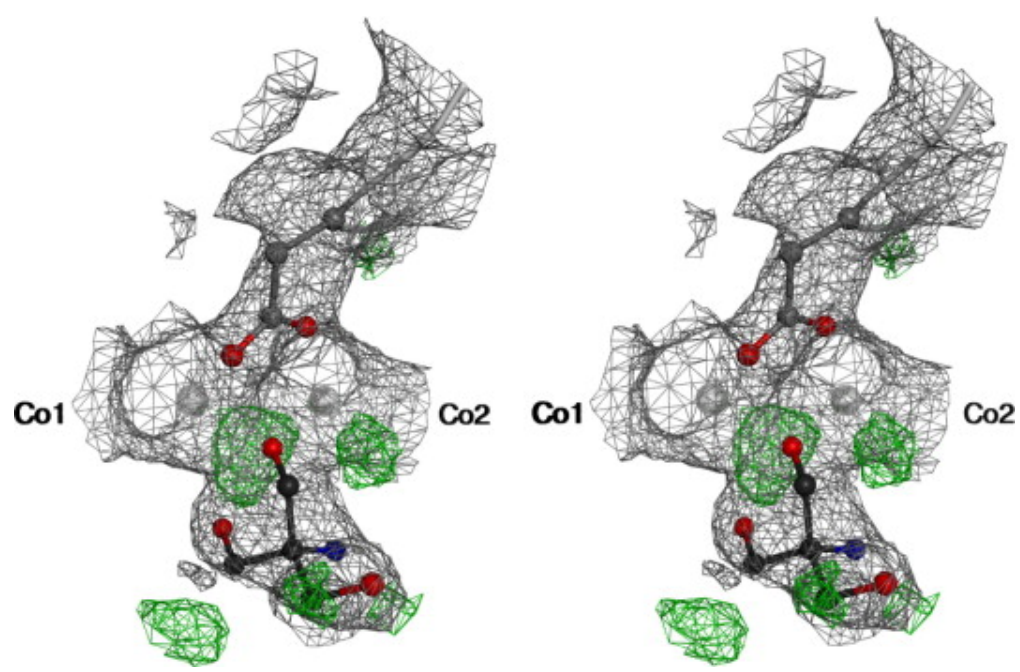

Fig. 3. A wall-eye stereo view of the [CoCo(AAP)] active site depicting Asp117 (in grey), the single Tris molecule (in black), and the two Co(II) ions (in chrome). Electron density maps are rendered with coefficients $2 F_{0}-F_{\mathrm{c}}$ (in grey) and $F_{0}-F_{\mathrm{c}}$ (in green) at 1.0 and $2.5 \sigma$, respectively. (For interpretation of the references to color in this figure legend, the reader is referred to the web version of this article.)

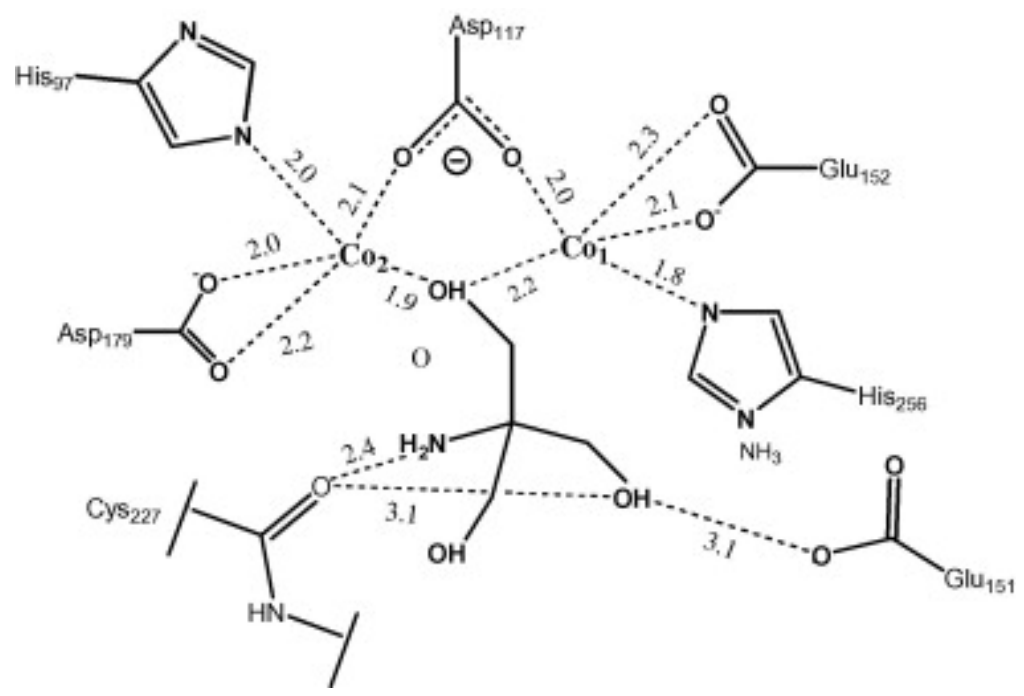

Fig. 4. Schematic of the [CoCo(AAP)]-Tris active site with distances shown in $\AA$. The binding mode of Tris is shown. The bridging oxygen is $\mathrm{O} 4$ and the $\mathrm{O} 5$ oxygen of Tris interacts with Glu 151 and the carbonyl oxygen of Cys 227.

Several hydrogen-bonding interactions also exist involving Tris, which likely stabilize the Tris binding mode. Perhaps most notably, there is at least one interaction with the carboxylate oxygen atoms of Glu151. Glu151 was recently shown to be essential for catalysis and functions as a general acid/base during catalysis [31]. Both oxygen atoms of Glu151 are too far from the metal-bound Tris hydroxyl oxygen atom (O4) to form a hydrogen bond (3.9 and 4.4 A) [32]. Both oxygen atoms of Glu151 are also too far from the Tris amine nitrogen (N1) since they reside 4.2 and $5.1 \AA$ away. However, Glu151 forms a hydrogen bond (3.1 $\AA$ ) with one of the unliganded hydroxyl oxygen atoms of Tris (05). Two additional hydrogen-bonding interactions exist, with the first occurring between this same Tris hydroxyl oxygen atom (05) and the carbonyl oxygen atom of Cys 227 (3.1 $\AA$ ), while the second occurs between the Tris amine nitrogen atom (N1) and the same carbonyl oxygen atom ( $2.4 \AA$ Å). 


\section{Discussion}

All of the crystallographic studies on AAP to date have been done on the native $\mathrm{Zn}$ (II)-loaded form of the enzyme ([ZnZn(AAP)]), however, most of the spectroscopic data reported for AAP have been collected on the Co(II)substituted forms ([ZnCo(AAAP)], [CoZn(AAP)], and [CoCo(AAP)]) [33], [34], [35]. The obvious assumption in analyzing the reported spectroscopic data being, that all of these forms are structurally and mechanistically equal. Inspection of the available literature, however, reveals some discrepancies between the Zn(II) X-ray crystallographic data and the Co(II) spectroscopic results suggesting that this assumption may not necessarily be accurate. In the native structure of AAP [25], Zn1 exhibits a distorted tetrahedral or TBP geometry with the $N^{\varepsilon}$ nitrogen of His 256 and the bridging water/hydroxide oxygen atom making up the axial positions of a TBP. On the other hand, $\mathrm{Zn} 2$ resides in a distorted tetrahedron or TBP geometry with the $N^{\varepsilon}$ nitrogen of His97 and the bridging water/hydroxide oxygen making up the axial positions of a TBP. Glu152 is coordinated in an asymmetric bidentate fashion to $\mathrm{Zn} 1$ with the additional oxygen atom providing a potential fifth ligand at a distance of $2.4 \AA$. Similarly, Asp179 is coordinated to $\mathrm{Zn} 2$ in an asymmetric bidentate fashion with the second oxygen atom residing $2.3 \AA$ from the metal center providing a potential fifth ligand. The $\mathrm{M}-\mathrm{M}$ distance is $3.5 \AA$ with water/hydroxide oxygen atom distances of $2.3 \AA$ from both $\mathrm{Zn} 1$ and $\mathrm{Zn} 2$. A hydrogen bond exists between Glu151 and the bridging water/hydroxide with a distance of $3.4 \AA$.

Previously reported electronic absorption spectra of AAP in the presence of one and two equivalents of Co(II) ([Co_(AAP)] and [CoCo(AAP)]) at pH 7.5 were interpreted based on the [ZnZn(AAP)] X-ray crystallographic data [13], [33]. For [Co_(AAP)], a visible absorption spectrum with a band at $525 \mathrm{~nm}\left(\varepsilon_{525} \sim 50 \mathrm{M}^{-1} \mathrm{~cm}^{-1}\right)$ is observed. Addition of a second equivalent of Co(II) increases the absorption intensity at $525 \mathrm{~nm}$ to $\sim 90 \mathrm{M}^{-1} \mathrm{~cm}^{-1}$. The addition of more than two equivalents of $\mathrm{Co}$ (II) does not alter the intensity or position of the observed absorption band. The added absorptivity $\left(\Delta \varepsilon_{525}\right.$ of $\left.\sim 40 \mathrm{M}^{-1} \mathrm{~cm}^{-1}\right)$ appears to be due to a broad underlying protein absorption band that is not due to $\mathrm{Co}(\mathrm{II})$ absorption. A similar broad absorption band was observed for the Co(II)-substituted $R_{2}$ subunit of ribonucleotide reductase, and is also evident in several Co(II) model complexes [36]. Therefore, the increase in absorption at $525 \mathrm{~nm}$ due to the second Co(II) ion is only $\sim 20 \mathrm{M}^{-1} \mathrm{~cm}^{-1}$. Ligand-field theory predicts that the first $\mathrm{Co}(\mathrm{II})$ bound to AAP resides in a severely distorted tetrahedral or a distorted TBP coordination environment while the second Co(II) ion is likely octahedral [13]. These conclusions were also supported by magnetic circular dichroism spectra [13].

EPR spectra have also been recorded for [Co_(AAP)] and [CoCo(AAP)] at pH 7.5 [33]. [Co_(AAP)] provides a distinctive EPR signal in which the ${ }^{59} \mathrm{Co}$ hyperfine pattern is not centered on the low-field absorption that could only be simulated as two species [33]. The first species exhibited an axial signal with $g_{\text {eff }}$ values of $5.75,4.50$, and 2.50. These values correspond to an $M_{s}=| \pm 1 / 2\rangle$ ground state transition with $g_{\text {real }}=2.57$ and $E / D=0.08$. The second species had $g_{\text {eff }}$ values of $6.83,2.95$, and 1.96 and exhibited a characteristic eight-line ${ }^{59}$ Co hyperfine pattern with $A_{z}=7.2 \mathrm{mT} ; g_{\text {real }}=2.57$; and $E / D=0.28$. On the other hand, the EPR spectrum of [CoCo(AAP)] reveals a very broad, nondescript signal that was simulated as a single axial species with $g_{\text {eff }}$ values of 5.10, 3.84, and 2.18; $M_{s}=| \pm 1 / 2\rangle ; g_{\text {real }}=2.25 ; E / D=0.1$ [33]. The intensity of this signal corresponds to 0.13 spins per mole of Co(II) indicating that the two $\mathrm{Co}(\mathrm{II})$ ions in AAP experience significant spin-spin interaction. Perpendicular mode EPR titration of apo-AAP with Co(II) revealed a low-field signal extending out of zero-field in samples with more than one equivalent of $\mathrm{Co}$ (II) added. Coincident with the appearance of this low-field perpendicular mode signal was the appearance of a parallel mode EPR signal with $g \sim 12$. These data indicate that the two high-spin $S=3 / 2 \mathrm{Co}(\mathrm{II})$ ions in the dinuclear active site of AAP are ferromagnetically coupled. Bennett and Holz [33] also demonstrated that metal binding to apo-AAP occurs in a sequential fashion. Moreover, it was shown, based on electronic absorption and EPR spectroscopy, that the spectroscopic signatures for [Co_(AAP)] and [CoZn(AAP)] were identical but distinct from those of [ZnCo(AAP)] [34].

Based solely on EPR data, the coordination number and geometry of the Co(II) ions in [Co_(AAP)] and [CoCo(AAP)] cannot be determined. However, some structural information can be gleaned from these data when combined with electronic absorption data. Therefore, the two species observed in the EPR spectrum of 
[Co_(AAP)] appear to be the result of constraints imposed on the Co(II) ion coordination geometry by the ligandfield. For example, the signal exhibiting resolvable ${ }^{59} \mathrm{Co}$ hyperfine splitting is likely due to a Co(II) ion in a highly constrained and asymmetric coordination geometry and not significantly broadened by factors such as $g$-strain that arise from microdistributions in the Co(II) coordination environment. In contrast, the second species and the EPR signal observed for [CoCo(AAP)] appear to be due to Co(II) centers with much less constraint upon their geometry. Such an environment is likely the result of the relaxation of ligand sphere-derived constraints consistent with the observation of a wider distribution of microheterogeneous structures in a given sample. It is reasonable to expect that when the distribution of microheterogeneous environments is relatively wide, the resultant $g$-strain in the EPR spectrum will preclude the observation of ${ }^{59} \mathrm{Co}$ hyperfine splitting, although the hyperfine interaction is still present. The combination of all of these data suggested the active site of Co(II)substituted AAP contains a bis( $\mu$-hydroxo/aquo)( $\mu$-carboxylato)dicobalt core with Co1 residing in a TBP geometry while $\mathrm{Co} 2$ is octahedral.

The structure of [CoCo(AAP)] coordinated by Tris confirms the structure proposed from spectroscopic measurements. The Co(II) ions in Co(II)-substituted AAP bind to the enzyme active site in a manner similar to $\mathrm{Zn}(\mathrm{II})$, indicating that the spectroscopic and kinetic studies conducted on Co(II)-substituted AAP were conducted on a form of AAP structurally similar to the native [ZnZn(AAP)]. There is still the mechanistic difference due to the metal characteristics to consider (i.e., the fact that the Co(II)-substituted enzyme is hyperactive with respect to the native form [13]), but the structural findings are applicable to both, and consistent between these different methods. The Co(II) ion bound to His256, which has been identified as Co1, clearly resides in a TBP geometry while the second $\mathrm{Co}(\mathrm{II})$ ion ( $\mathrm{CO} 2)$ also resides in a distorted TBP geometry, contrary to electronic absorption data. Although few active site water molecules are observed in the X-ray structure of [CoCo(AAP)]Tris, a second hydroxyl group of Tris has access to Co2 suggesting that this $\mathrm{Co}(\mathrm{II})$ ion can expand its coordination geometry by binding a water molecule and become octahedral. Such an equilibrium would be consistent with the observed spectroscopic data. Interestingly, in [CoCo(AAP)], the first as well as the second carboxylate oxygens of the active site metal ligands Glu152 and Asp179 appear to be, on average, closer to the metal than in [ZnZn(AAP)] complexes, which would be consistent with the preference of Co(II) relative to $\mathrm{Zn}(\mathrm{II})$ to be octahedral. However, at the current resolution of the structure, the estimated coordinate error is $\pm 0.3 \AA$, so the difference cannot be considered significant.

The X-ray crystal structure of [ZnZn(AAP)] bound by Tris was recently solved to $1.2 \AA[30]$ and shows both similarities and differences as compared to the structure of [CoCo(AAP)]-Tris. Although the intermetal distance of $3.3 \AA$ in the Co(II)-loaded Tris complex is $0.2 \AA$ shorter than that for the $\mathrm{Zn}$ (II)-loaded one, this value is probably not significant at the resolution of the cobalt-substituted structure. In the zinc Tris structure, the metal-endogenous ligand coordination distances are an average representation of two exogenous ligands (Tris, $\mathrm{H}_{2} \mathrm{O}$ ) binding modes. For [ $\mathrm{nnZn}(A A P)$ ], there is reportedly an $80-20$ ratio of Tris vs. $\mathrm{H}_{2} \mathrm{O} / \mathrm{OH}^{-}$binding observed in the active site [30], which would loosely imply an equilibrium between TBP and a distorted tetrahedral geometry on each of the $\mathrm{Zn}$ cations. Our current interpretation of the binding of Tris to the cobalt form of the enzyme is similar: an average of a single Tris binding mode plus water-substituted dimetal center. In accord with the preference of $\mathrm{Co}$ (II) for coordination numbers higher than $\mathrm{Zn}(\mathrm{II})$, its coordination spheres appear somewhat tighter. Most notably, with Co1 having only a single exogenous ligand interaction, the Co1-His 256 coordination distance is $1.8 \AA$, significantly shorter than seen in any of the [ZnZn(AAP)] complexes (Table 2). Similarly, the dangling oxygens of Glu152 and Asp179 are slightly further from the metal in the Zn-loaded complex. However, due to the estimated coordinate error of $\pm 0.3 \AA$ for the Co-loaded structure, this difference, again, cannot be considered significant.

In the [ZnZn(AAP)]-Tris structure, Tris coordinates to the two metal ions by interactions with three of its functional groups (Fig. 5). A hydroxyl oxygen atom of Tris (O4) bridges the two $\mathrm{Zn}(\mathrm{II})$ ions in the position where a bridging water species might be expected, similar to the binding mode observed for [CoCo(AAP)]-Tris (Fig. 3, Fig. 4), with a Zn1-O distance of $2.2 \AA$ and a Zn2-O distance of $2.0 \AA$. . A second hydroxyl oxygen atom (O5) 
coordinates to $\mathrm{Zn} 2$ with a distance of $2.2 \AA$, which is not observed in the binding of Tris to [CoCo(AAP)]. Also in contrast with the observed Tris binding mode for [CoCo(AAP)], the free amine of Tris (N1) coordinates to Zn1 in the $[\mathrm{ZnZn}(\mathrm{AAP})]-$ Tris structure with a $\mathrm{Zn}-\mathrm{N}$ distance of $2.2 \AA$. The final hydroxyl oxygen atom (06) of Tris forms a hydrogen bond with the phenolic oxygen atom of Tyr225 in the zinc enzyme, but this interaction is too long in the cobalt enzyme, although there is some flexibility at this end of the bound Tris in that structure.

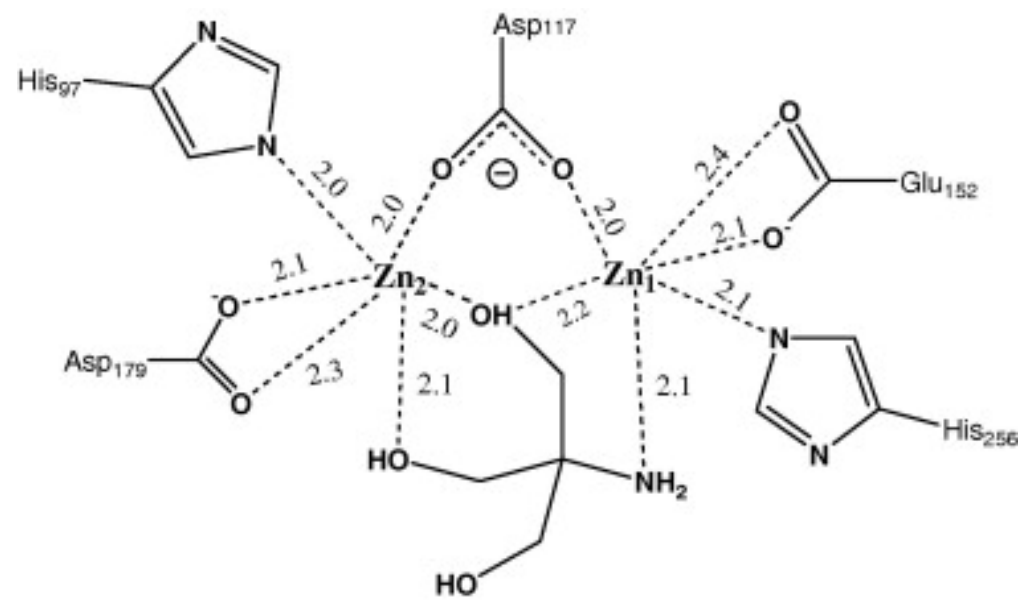

Fig. 5. Schematic of the active site of the [ZnZn(AAP)]-Tris [30] complex with distances shown in $\AA$.

Considering the relatively small size of the Tris molecule, it appears to have a large degree of freedom to interact with the dinuclear active site of AAP. That Tris can bind to AAP in one of several ways is exemplified by the observed X-ray crystal structures of both Zn(II)- and Co(II)-loaded AAP (Fig. 4, Fig. 5). One of the major deciding factors determining the mode of ligand coordination, given that $\mathrm{pH}$ and ionic strength were identical for both crystallization solutions, would seem to be the identity of the divalent metal ions in the active site. The choice of metal cation would differently affect the $\mathrm{p} K_{\mathrm{a}}$ of any coordinating ligands as well as determine the preferred coordination geometry. Thus the observed decrease in the $\mathrm{Co}-\mathrm{O}$ bond distances for the dangling oxygen atoms of Glu152 and Asp179, relative to the [ZnZn(AAP)]-Tris structure, is indicative of the Co(II) preference for TBP or octahedral coordination geometries. In the binding of Tris to [CoCo(AAP)], $\mathrm{O} 4$ forms a single oxygen bridge between the metals. The same can be found in the [ZnZn(AAP)]-Tris complex [30]. For the cobalt enzyme this is the only Tris atom coordinated to the metals. The Tris $\mathrm{O} 4$ resides within hydrogen-bonding distance of both Asp117 carboxylate oxygens and in geometry favorable to hydrogen bond formation with OD1. Such an interaction would decrease the negative charge on this Asp117 oxygen and hence slightly elongate its distance from $\mathrm{Co} 2$, relative to the distance of OD2 from $\mathrm{Co} 2$. On the other hand, the coordination of the now more negatively charged Tris $\mathrm{O} 4$ would be expected to be tighter (1.9 $\AA$ ) to $\mathrm{Co} 2$ than to Co1 ( $2.2 \AA)$. The partial deprotonation of the $\mathrm{O} 4$ oxygen would also allow for overall stronger coordination to the bimetal core than if the hydrogen-bonding interaction were not present.

In both cases, there is also a possibility for a strong interaction with the OE1 of Glu152. However, this being the more strongly metal-coordinating oxygen of Glu152, a hydrogen bond with either of the expectedly more basic Glu151 oxygens appears much more likely. At an O-O distance of $2.7 \AA$, this would indicate a very strong hydrogen bond, where either of the involved oxygens might be protonated, and would thus be supportive of the general acid/base role that Glu151 has been proposed to play at the various stages of the reaction mechanism [31] [38]. Based on the coordination distances of $\mathrm{O} 4$ from the metals, one could postulate $\mathrm{O} 4$ to be protonated in the Co-loaded complex and deprotonated in the $\mathrm{Zn}$-loaded one. Similarly, the interaction of 05 in the [ZnZn(AAP)]-Tris complex with the OD1 oxygen of Asp117, which appears favorable to hydrogen bond formation, could further facilitate coordination of 05 to $\mathrm{Zn} 2$ that is tighter than what is seen in the Co-loaded complex. Due to the small size of the Tris molecule its binding mode to the Co-loaded AAP appears to be strictly competitive due to metal ion chelation rather than in some way mechanistically related. 


\section{Supplementary material}

The coordinates have been deposited at the Protein Data Bank and are available under the access code 2PRQ.

\section{Abbreviations}

$\begin{array}{ll}\text { HEPES } & \text { [4-(2-hydroxyethyl)-1-piperazineethanesulfonic acid] } \\ \text { Tricine } & N \text {-Tris[hydroxymethyl]methylglycine } \\ \text { Tris } & \text { Tris(hydroxymethyl)aminomethane } \\ \text { I-pNA } & \text { I-leucine } p \text {-nitroanilide } \\ \text { Bestatin } & {[(2 S, 3 R) \text {-3-amino-2-hydroxy-4-phenylbutanoy]-leucine }} \\ \text { LPA } & \text { I-Leucinephosphonic acid } \\ \text { BuBA } & \text { 1-butaneboronic acid } \\ \text { AAP } & \text { aminopeptidase from Aeromonas proteolytica } \\ \text { bILAP } & \text { bovine lens leucine aminopeptidase } \\ \text { o-phen } & \text { 1,10-phenanthroline } \\ \text { UV-visible } & \text { electronic absorption } \\ \text { EPR } & \text { electron paramagnetic resonance }\end{array}$

\section{Acknowledgements}

This work was supported by the National Science Foundation (CHE-0652981, RCH), the Medical College of Wisconsin Research Affairs Committee (BB), the National Institutes of Health (GM26788, GAP and DR), and in part, by the Macromolecular Structure and Mechanisms Training Grant from NIH (CCS). The Bruker ESP-300E EPR spectrometer was purchased with funds provided by the National Science Foundation (BIR-9413530) and XSophe was purchased with funds from the National Institutes of Health (NIH RR01008, BB).

\section{References}

[1] W.N. Lipscomb, N. Sträter Chem. Rev., 96 (1996), pp. 2375-2433

[2] D.E. Wilcox Chem. Rev., 96 (1996), pp. 2435-2458

[3] G.C. Dismukes Chem. Rev., 96 (1996), pp. 2909-2926

[4] R.P. Hausinger E. Frieden (Ed.), Biochemistry of Nickel, vol. 12, Plenum Press, New York (1993)

[5] J. Chin Acc. Chem. Res., 24 (1991), pp. 145-152

[6] K. Lai, K.I. Dave, J.R. Wild J. Biol. Chem., 269 (1994), pp. 16579-16584

[7] F.M. Menger, L.H. Gan, E. Johnson, D.H. Durst J. Am. Chem. Soc., 109 (1987), pp. 2800-2803

[8] G. Pulido-Cejudo, B. Conway, P. Proulx, R. Brown, C.A. Izaguirre Antivir. Res., 36 (1997), pp. 167-177

[9] I. Bertini, C. Luchinat Adv. Inorg. Biochem., 6 (1984), pp. 71-111

[10] I. Bertini, C. Luchinat, M. Rosi, A. Sgamellotti, F. Tarantelli Inorg. Chem., 29 (1990), pp. 1460-1463

[11] F.A. Cotton, G. Wilkinson, C.A. Murillo, M. Bochmann Advanced Inorganic Chemistry John Wiley \& Sons, New York (1999)

[12] J.M. Prescott, F.W. Wagner, B. Holmquist, B.L. Vallee Biochem Biophys. Res. Commun., 114 (1983), pp. 646652

[13] J.M. Prescott, F.W. Wagner, B. Holmquist, B.L. Vallee Biochemistry, 24 (1985), pp. 5350-5356

[14] R.H. Holm, P. Kennepohl, E.I. Solomon Chem. Rev., 96 (1996), pp. 2239-2314

[15] N. Sträter, W.N. Lipscomb, T. Klabunde, B. Krebs Angew. Chem. Int. Ed. Engl., 35 (1996), pp. 2024-2055

[16] W.T. Lowther, B.W. Matthews Chem. Rev., 102 (2002), pp. 4581-4607

[17] B.L. Vallee, D.S. Auld Proc. Natl. Acad. Sci. USA, 90 (1993), pp. 2715-2718

[18] J.M. Prescott, S.H. Wilkes Methods Enzymol., 45 (1976), pp. 530-543

[19] G. Chen, T. Edwards, V.M. D'souza, R.C. Holz Biochemistry, 36 (1997), pp. 4278-4286

[20] J.O. Baker, S.H. Wilkes, M.E. Bayliss, J.M. Prescott Biochemistry, 22 (1983), pp. 2098-2103

[21] J.M. Prescott, S.H. Wilkes, F.W. Wagner, K.J. Wilson J. Biol. Chem., 246 (1971), pp. 1756-1764 
[22] H. Edelhoch Biochemistry, 6 (1967), pp. 1948-1954

[23] S.C. Gill, P.H. von Hippel Anal. Biochem., 182 (1989), pp. 319-326

[24] S.C. Gill, T.D. Yager, P.H. von Hippel Biophys. Chem., 37 (1990), pp. 239-250

[25] B. Chevrier, C. Schalk, H. D'Orchymont, J.-M. Rondeau, D. Moras, C. Tarnus Structure, 2 (1994), pp. 283-291

[26] Z. Otwinowski, W. Minor Methods Enzymol., 276 (1997), p. 307

[27] A.T. Brünger X-PLOR, A System for X-ray Crystallography and NMR (version 3.851) Yale University Press, New Haven, CT (1997)

[28] C. DePaola, B. Bennett, R.C. Holz, D. Ringe, G. Petsko Biochemistry, 38 (1999), pp. 9048-9053

[29] C. Stamper, B. Bennett, T. Edwards, R.C. Holz, D. Ringe, G. Petsko Biochemistry, 40 (2001), pp. 7034-7046

[30] W. Desmarais, L.D. Bienvenue, K. Bzymek, R.C. Holz, A.G. Petsko, D. Ringe Structure (2002), pp. 1063-1072

[31] K.P. Bzymek, R.C. Holz J. Biol. Chem., 279 (2004), pp. 31018-31025

[32] D.W. Christianson, R.S. Alexander J. Am. Chem. Soc., 111 (1989), pp. 6412-6419

[33] B. Bennett, R.C. Holz J Am. Chem. Soc., 119 (1997), pp. 1923-1933

[34] B. Bennett, R.C. Holz Biochemistry, 36 (1997), pp. 9837-9846

[35] B. Bennett, R.C. Holz J. Am. Chem. Soc., 120 (1998), pp. 12139-12140

[36] T.E. Elgren, L.-J. Ming, L. Que Inorg. Chem., 33 (1994), pp. 891-894

[37] C.C. Stamper, D.L. Bienvenue, B. Bennett, D. Ringe, G.A. Petsko, R.C. Holz Biochemistry, 43 (2004), pp. $9620-$ 9628

[38] G. Schürer, H. Lanig, T. Clark Biochemistry, 43 (2004), pp. 5414-5427 